\title{
POSITRON EMISSION TOMOGRAPHY: USE OF SHORT-LIVED RADIONUCLIDES FOR NEUROLOGICAL RESEARCH
}

\author{
R.D. HICHWA \\ University of Michigan Medical School, Cyclotron/PET Facility *, Ann Arbor, MI 48109, USA
}

Positron emission tomography (PET) measures the temporal and spatial distributions of short-lived positron emitting nuclides within the human body. Positron annihilation radiation is detected by rings of discrete scintillators surrounding the body. Images $(128 \times 128$ pixels $)$ of the radioactivity distributions are reconstructed from the discrete ray projections of the detected coincident photons. Each pixel is fit to a mathematical model that represents a physiological process such as blood flow or metabolism to create a new three parameter functional or parametric image.

\section{Introduction}

Positron Emission Tomography or PET is a rapidly advancing multidisciplinary field that utilizes the onsite production of short-lived positron emitting nuclides with a cyclotron or other particle accelerator such as a tandem Van de Graaff. Human physiology and, in our particular case, brain physiology is measured by observing the annihilation of these positron emitters from biologically compatible radiopharmaceuticals [1-3]. The detection of the annihilation radiation (two diametrically opposed $511 \mathrm{keV}$ photons) in a PET scanner allows an image to be created that represents the spatial distribution of the radiotracer [4]. The acquisition time for each data set varies. The static distribution for various radiopharmaceuticals after an incorporation period is proportional to some physiological phenomenon and it may require 10 to $20 \mathrm{~min}$ to acquire sufficient statistics for meaningful images. A F-18 labeled glucose analog follows this behavior and is used to map the metabolic activity within the brain [5]. Since ${ }^{18}$ F-FDG is not normally found in the body but behaves much like glucose, the brain's normal metabolic fuel, it is a perfect radioactive tracer [6]. Other agents such as ${ }^{15} \mathrm{O}-\mathrm{H}_{2} \mathrm{O}$ require rapid temporal sampling of the kinetic behavior of the radiolabeled tracer in order to measure, for instance, cerebral blood flow. In this case, five second data sets are acquired for approximately two minutes to measure the kinetic uptake and release from the brain tissue. Each pixel in the 24 frame study is fit to a physiological model. Blood flow is one parameter in that model. A second image is created that substitutes the calculated flow parameter into the $x-y$ pixel location originally used for the fitting procedure. The new

* Work supported by NINCDS 2P01 NS15655-06. image is called a functional or parametric image. The following pages outline in more detail the facilities applicable to PET research and the current methods employed for PET data acquisition and analysis.

\section{PET facilities}

The University of Michigan Cyclotron/PET Facility performs three major functions that utilize approximately 7500 square feet of space. The cyclotron/target systems, the radiochemical/radiopharmaceutical lab and the PET tomograph with associated computers for data reduction and image processing comprise the Facility laboratories.

\subsection{Cyclotron}

The Cyclotron Corporation (TCC) CS-30 accelerator [7] is a four particle, fixed energy, positive ion, isochronous cyclotron. Protons, deuterons, ${ }^{3} \mathrm{He}^{2+}$, and ${ }^{4} \mathrm{He}^{2+}$ ions produced with a Penning ion gauge source are accelerated to energies of $26 \mathrm{MeV}, 15 \mathrm{MeV}, 38 \mathrm{MeV}$ and $30 \mathrm{MeV}$ respectively. The extraction radius is $42 \mathrm{~cm}$ for the $96.5 \mathrm{~cm}$ diameter sector focusing, $17.5 \mathrm{kG}$ magnet. Particles are accelerated in the fundamental mode with a stable, self-exiting RF system to facilitate high efficiency precessional extraction. An electrostatic deflector and septum located at the outside edge of the magnet pole are used for beam extraction. Measured internal beam currents for the four particles above are $200,300,135$, and $90 \mu \mathrm{A}$ respectively and measured external currents are $60,100,60$ and $40 \mu \mathrm{A}$ respectively.

Three improvements are planned to increase the usable beam current while reducing activation of machine components. Movable slits near the central region will delineate small radius orbits thereby reducing un- 
Table 1

Beam splitting magnet specifications

\begin{tabular}{ll}
\hline Bend angle & $5^{\circ}(0.087 \mathrm{rad})$ \\
Beam stiffness & $292 \mathrm{kG}$ in \\
Beam energy (protons) & $26 \mathrm{MeV}$ \\
Magnetic field & $1.7 \mathrm{kG}$ \\
Effective length & $15 \mathrm{in}$ \\
Ampere turns & $5147 \mathrm{~A}$ turns \\
Gap height & $1.5 \mathrm{in}$ \\
Number turns & 4 \\
Magnet current & $1286 \mathrm{~A}$ \\
Power supply excitation & $\mathrm{DC}$ \\
Power supply required & $1400 \mathrm{~A}$ \\
\hline
\end{tabular}

wanted high energy scattered radiation. A diagnostic probe at or near the extraction region will permit tuning of the harmonic coils, deflector position, deflector voltage and magnetic channel to optimize extraction efficiency. This probe will consist of a rotating wire scanner with $x-y$ display. Lastly, a regulated ion source supply will be installed to extend the life of the Penning cathodes. Other radiation detection probes (n-gamma) may be used to assist in the tuning procedures.

A beam splitting magnet is to be constructed to permit simultaneous irradiation of three targets. RF synchronized multiplexing of the beam was rejected due to duty cycle and maximum target current limitations. Instead, a design that implements two remotely positioned, high power, septum magnets similar to the design used on the LBL Super-HILAC [8] was chosen. With this method, the intensity of the individual beams will be reduced but the $100 \%$ duty cycle on target will be preserved. Existing and proposed target systems do not utilize all available extracted beam current. Splitting the total cyclotron beam output better capitalizes on the CS-30 performance parameters. Design specifications for the splitting magnet are given in table 1 . Motors are used to position the septum magnets creating one, two or three derived beams from the ion beam entering the magnet. The entire assembly will be mounted in a vacuum box upstream from the existing $\pm 15^{\circ}$ switching magnet. Shielding about the magnet box will reduce high radiation levels expected from induced activation of the septum magnet and related components.

\subsection{Targetry}

There are six target systems currently in operation. The first is an internal, pneumatically operated, medium power $(5 \mathrm{~kW})$ solid target system. Simple sequencer technology is used to manipulate vacuum integrity, cooling, target orientation and longitudinal position of the target.

Future developments with this system include modification to permit high power, gas target operation for steady-state production of either $0-15$ or C-11.

All remaining targets are external to the cyclotron. The simplest targets are cylindrical and water cooled for the continuous production of $\mathrm{C}-11$ and $\mathrm{O}-15$. An insertable water target is used for N-13. A gaseous fluorine target is used to produce $F-18 F_{2}$. An external, low power $(1.5 \mathrm{~kW})$, pneumatically operated, solid target is used for irradiation of powders, metals or vacuum deposited materials. Table 2 summarizes common target operations.

\subsection{Radiochemistry laboratory}

Routine syntheses and experimental radiopharmaceutical development are performed in a 1100 square foot laboratory. Pipelines conduct the radioactive target gases and liquids into a shielded hot cell. The agents primarily used for PET scanning and their typical injected or inhaled doses are as follows: $\left[{ }^{15} \mathrm{O}\right]-\mathrm{CO}$ $(50 \mathrm{mCi}),\left[{ }^{15} \mathrm{O}\right]-\mathrm{CO}_{2}(50 \mathrm{mCi}),\left[{ }^{11} \mathrm{C}\right]-\mathrm{CO}(30 \mathrm{mCi})$, $\left[{ }^{11} \mathrm{C}\right]-\mathrm{CO}_{2}(30 \mathrm{mCi}),\left[{ }^{15} \mathrm{O}\right]-\mathrm{O}_{2}(50 \mathrm{mCi}),\left[{ }^{15} \mathrm{O}\right]-\mathrm{H}_{2} \mathrm{O}$ $(50 \mathrm{mCi}),\left[{ }^{18} \mathrm{~F}\right]-2$-fluoro-2-deoxy-D-glucose $(10 \mathrm{mCi})$ and $\left[{ }^{13} \mathrm{~N}\right]-\mathrm{NH}_{3}(20 \mathrm{mCi})$. The laboratory maintains complete radio-HPLC and radio-GC systems to test for chemical, radiochemical and nuclidic purities as well as for development of new agents.

\subsection{Tomography scanner}

PET data is acquired with the TCC PCT-4600A positron tomograph. This is a three ring, five slice machine. Each ring consists of 96 bismuth germanate (BGO) detectors, $1.96 \mathrm{~cm}$ in diameter by $3.8 \mathrm{~cm}$ long. The in-plane resolution is $10.9 \mathrm{~mm}$ fwhm which indicates the apparent size of a point radiation source. This

Table 2

\begin{tabular}{llllll}
\hline Target & Nuclide & Half-life & Reaction & Beam current & Target parameters ${ }^{a)}$ \\
\hline Alpha & O-15 & $122 \mathrm{~s}$ & ${ }^{14} \mathrm{~N}(\mathrm{~d}, \mathrm{n})^{15} \mathrm{O}$ & $10 \mu \mathrm{A}$ & $E_{\text {in }}=8 \mathrm{MeV}, P=60 \mathrm{psi}, V=36 \mathrm{~cm}^{3}, T=S \mathrm{~S}$ \\
Beta & $\mathrm{C}-11$ & $20.4 \mathrm{~m}$ & ${ }^{14} \mathrm{~N}(\mathrm{p}, \alpha)^{11} \mathrm{C}$ & $5-10 \mu \mathrm{A}$ & $E_{\text {in }}=12 \mathrm{MeV}, P=155 \mathrm{psi}, V=30, T=S S$ \\
Water & $\mathrm{N}-13$ & $9.97 \mathrm{~m}$ & ${ }^{16} \mathrm{O}(\mathrm{p}, \alpha)^{13} \mathrm{~N}$ & $5-10 \mu \mathrm{A}$ & $E_{\text {in }}=18 \mathrm{MeV}, P=1 \mathrm{~atm}, V=1.0 \mathrm{~cm}^{3}, T=5 \mathrm{~min}$ \\
$F_{2}$ & $\mathrm{~F}-18$ & $109.8 \mathrm{~m}$ & ${ }^{20} \mathrm{Ne}(\mathrm{d}, \alpha)^{18} \mathrm{~F}$ & $10 \mu \mathrm{A}$ & $E_{\text {in }}=14.5 \mathrm{MeV}, P=94 \mathrm{psi}, V=56 \mathrm{~cm}^{3}, T=40 \mathrm{~min}$ \\
\hline
\end{tabular}

A) $E_{\text {in }}=$ incident energy, $P=$ target pressure, $V=$ target volume, $T=$ typical irradiation time, SS $=$ steady state. 
resolution is degraded to $13.0 \mathrm{~cm}$ at a $10 \mathrm{~cm}$ radius from the axis through the center of the scanner (longitudinal axis). Sparse data sampling at this radius accounts for the resolution reduction. Resolution along the longitudinal axis is $8.0 \mathrm{~mm}$ fwhm. Slice separation is $1.1 \mathrm{~cm}$ from center-to-center. The scanner sensitivity is $24000 \mathrm{cts} /$ $\mathrm{s} / \mu \mathrm{Ci} / \mathrm{cm}^{3}$ in a $20 \mathrm{~cm}$ diameter homogeneous phantom. The maximum count rate capability defined as the rate in which the random count rate equals the true count rate is $3.1 \times 10^{4} \mathrm{cts} / \mathrm{s}$. Virtually all data are acquired in the high resolution mode. A minimum scan time of $5.0 \mathrm{~s}$ is needed to complete the wobble cycle for this mode. Wobble is used to increase the total number of rays that comprise a data set. The motion of the detector ring as viewed end-on is oscillatory with a period of $5.0 \mathrm{~s}$. Each physical detector can then be thought of as composed of eight equally spaced but smaller diameter detectors. Simple rotation about the longitudinal axis would achieve similar results. The engineering constraints of maintaining the electrical cabling to 288 photomultiplier/discriminator channels is more easily handled by wobbling the detector rings with an eccentricity of only $1.0 \mathrm{~cm}$.

\subsection{Image processing laboratory}

Parallel ray-angle data from the PCT-4600A is buffered and ported to a DEC LSI-11/23 [9] for storage. A DEC PDP-11/44 is used to reconstruct and display data. An Analogic AP-400 array processor [10] provides enhanced image manipulation speeds so that a high resolution data set is reconstructed into $128 \times 128$ pixel images in $5 \mathrm{~s}$. A DeAnza ID-2000 image display processor [11] is used to view reconstructed images for proper orientation, choice of filter cut-off frequency and analytic attenuation corrections. A single data set that consists of seven image planes (one for each ring and two for crossplanes between rings) are combined into five image slices according to individual plane sensitivities. Region-of-interest analysis, thresholding, arithmetic operations and color coding of images are performed with a DEC Gamma-11 and a Computer Design and Applications Delta-11 [12] image processing system.

\section{Experimental procedures}

Patients and normal volunteers are selected according to protocols established for each project. Prior to the scanning procedure, all subjects receive a radial artery catheter in either arm and external landmarks (head diameter, distance from the top of the head to an imaginary line passing through the canthus of the eye to the meatus of the ear or $\mathrm{CM}$ line) are measured. $\mathrm{Pa}$ tients are then positioned in the scanner, supine, with their head oriented so that the plane passing through the CM line is parallel to the tomograph detector rings.

An intravenous bolus injection of the appropriate radiopharmaceutical is synchronized with the start of the tomograph and ancillary computers. Arterial blood samples are collected according to the type of study being performed. For ${ }^{18}$ F-FDG studies, discrete $1 \mathrm{ml}$ blood samples are collected at $5 \mathrm{~s}$ intervals for $2 \mathrm{~min}, 1$ min intervals for the next $5 \mathrm{~min}$ and $10 \mathrm{~min}$ intervals for the next $30 \mathrm{~min}$. Each sample is centrifuged to separate the plasma from the red blood cells. $250 \mu 1$ of plasma are withdrawn from each sample and the F-18 activity is counted in a well counter. A ${ }^{18} \mathrm{~F}$-FDG plasma curve is constructed from the decay corrected data. For ${ }^{15} \mathrm{O}-\mathrm{H}_{2} \mathrm{O}$ studies, arterial samples are continuously withdrawn at the rate of $7-14 \mathrm{ml} / \mathrm{min}$ for $2 \mathrm{~min}$ with a peristaltic pump. Spare scanner detectors operating in coincidence are used to measure the blood ${ }^{15} \mathrm{O}-\mathrm{H}_{2} \mathrm{O}$ concentration. An arterial input function curve is created once the mixing response of the sampling tubing and detector volumes are deconvolved from the blood data. Calibrations are performed between the well counter and the scanner as well as the continuous flow detectors and the scanner to permit the conversion of blood data into equivalent tomograph count rates.

The scan times have been discussed in section 1 for each of these agents. For ${ }^{18}$ F-FDG where the distribution of the glucose is static after the incorporation period, the patient is moved $0.5 \mathrm{~cm}$ out of the scanner by means of a motorized couch. A second scan is performed. The patient is again indexed $5.5 \mathrm{~cm}$ out of the sanner and the imaging process is repeated. In this way, 20 slices of brain ${ }^{18} \mathrm{~F}$-FDG activity $(11.0 \mathrm{~cm}$ total) are recorded on $0.55 \mathrm{~cm}$ centers in about $40-60 \mathrm{~min}$ from a single injection of ${ }^{18} \mathrm{~F}$-FDG. The same scanning procedure is performed for ${ }^{15} \mathrm{O}-\mathrm{H}_{2} \mathrm{O}$ but requires an injection for each set of five brain slices. After the scanning session, the arterial line is removed and the patients are free to leave the Facility suffering no ill-effects.

Images are reconstructed using a Hanning weighted ramp filter with a cut-off frequency of 1.4 cycles $/ \mathrm{cm}$. Images are corrected for half-life, random events, attenuation, wobble, deadtime and detector inhomogeneities [13].

Each set of five brain slices are decay corrected to the start of the scan. For random subtraction, singles data $\left(N_{1}, N_{2}\right)$ are collected for each detector in a coincidence pair. The coincidence resolving time $(\tau)$ is fixed in the hardware at 20 ns. Elements of the coincidence ray-angle data set are corrected by subtracting the random count rate $\left(N_{\mathrm{R}}\right)$ according to the following equation.

$N_{\mathrm{R}}=N_{1} N_{2} 2 T$.

An analytical attenuation correction is performed by 
creating an ellipse that closely approximates the dimensions of the cross-sectional brain slice. An effective attenuation coefficient $\left(0.085 \mathrm{~cm}^{-1}\right)$ for $511 \mathrm{keV}$ photons in water is used. The coincidence data for each plane are multiplied by a matrix of numerical values within the boundary of the ellipse to correct for the observed reduced count rates due to attenuation. A second method is about to be instituted in which a sealed rod source of Ge-68 activity is rotated around the patient's head. The actual attenuated count rates opposite the rod will be used to correct patient data.

A wobble correction is applied since each detector spends more time sampling the radioactivity distribution near the ends of its oscillatory excursion. This is a symmetrical correction since the motion from $0^{\circ}$ to $\pi$ is equivalent to the motion from $\pi$ to $2 \pi$. Each detector then is associated with eight bins with the following correction for each bin: $0.8372,0.8410,1.1564,1.3214$, $1.3214,1.1564,0.8410,0.8372$. These numbers reflect the actual mechanical wobble correction for the PCT. $4600 \mathrm{~A}$. Total coincidence counts are conserved.

Singles and coincidence count rates are corrected for deadtime losses. The corrections were determined empirically by injecting known random pulse rates into each section of the tomograph electronics and observing the count rate losses. It was found that the limiting areas were those in which singles data were merged onto multiplexed internal busses. Significant improvements in tomograph maximum count rates have been accomplished by reducing events from propagating through the system that are not valid ray-angle counts.

Each detector is automatically tuned for the maximum count rate by centering the photopeak with the energy discriminator. However, there is still a distribution of detector responses from a calibrated source. Occasionally, detectors register low count rates due to poor contact in one of thousands of socketed IC connections or just completely fail. To overcome this problem a calibration scan is performed that measures the response of each detector to the same Ge-68 rod source discussed earlier. The average count rate is calculated and those detectors that fall outside the acceptable limits from the norm are flagged. A correction mask is created and the raw data are multiplied by this mask. Tests are then run to measure the uniformity of the scanner response to a uniform, homogeneous distributed source.

The final images have all corrections applied. For ${ }^{18}$ F-FDG, each pixel in the corrected image is input to the deoxy-glucose utilization model given in the equation below and the local cerebral metabolic rate of glucose (LCMRG) is calculated. Some of the arterial blood samples also have serum glucose measured as well as ${ }^{18}$ F-FDG concentrations. The analytic attenuation mask is used to calculate only those LCMRG values within the brain. Table 3 defines each of the symbols in the glucose model.

$$
\begin{aligned}
\mathrm{LCMRG}= & C_{\mathrm{p}}\left(C_{i}^{*}(T)-\frac{D \cdot C A L k_{1}}{\alpha_{2}-\alpha_{1}}\left[\left(k_{4}-\alpha_{1}\right) \mathrm{e}^{-\alpha_{1} t}\right.\right. \\
& \left.\left.+\left(\alpha_{2}-k_{4}\right) \mathrm{e}^{-\alpha_{2} t}\right] \odot C p^{*}(t)\right) \\
& /\left(D \cdot C A L \cdot \mathrm{SG} \cdot \mathrm{LC} \frac{k_{2}+k_{3}}{\alpha_{2}-\alpha_{1}}\right. \\
& \left.\times\left[\mathrm{e}^{-\alpha_{1} t}-\mathrm{e}^{-\alpha_{2} t}\right] \odot C p^{*}(t)\right)
\end{aligned}
$$

where

$$
\begin{aligned}
\alpha_{1}= & \left\{\left(k_{2}+k_{3}+k_{4}\right)\right. \\
& \left.-\left[\left(k_{2}+k_{3}+k_{4}\right)^{2}-4 k_{2} k_{4}\right]^{1 / 2}\right\} / 2, \\
\alpha_{2}= & \left\{\left(k_{2}+k_{3}+k_{4}\right)\right. \\
& \left.+\left[\left(k_{2}+k_{3}+k_{4}\right)^{2}-4 k_{2} k_{4}\right]^{1 / 2}\right\} / 2 .
\end{aligned}
$$

Local cerebral blood flow (LCBF) values are calculated according to the model below and expressed as flow/unit volume.

\begin{tabular}{|c|c|}
\hline LCMRG & $\begin{array}{l}\text { local cerebral metabolic rate of glucose (mg/ } \\
\min / 100 \mathrm{~g} \text { tissue) }\end{array}$ \\
\hline$C_{p}$ & plasma glucose concentration ( $\mathrm{mg} / \mathrm{dl}$ ) \\
\hline$C_{\mathrm{i}}^{*}(T)$ & PET data (PET cts $/ \mathrm{min} /$ pixel) \\
\hline$C_{p}^{*}(t)$ & $\begin{array}{l}{ }^{18} \mathrm{~F} \text {-FDG plasma concentration (well cts/min/ } \\
\mathrm{ml} \text { ) }\end{array}$ \\
\hline LC & model lumped constant $(0.38)$ \\
\hline$k_{1}$ & $\begin{array}{l}\text { plasma to precursor forward grey matter rate } \\
\text { constant }\left(0.092 \mathrm{~min}^{-1}\right)\end{array}$ \\
\hline$k_{2}$ & $\begin{array}{l}\text { plasma to precursor reverse grey matter rate con- } \\
\text { stant }\left(0.210 \mathrm{~min}^{-1}\right)\end{array}$ \\
\hline$k_{3}$ & $\begin{array}{l}\text { precursor to metabolite forward grey matter rate } \\
\text { constant }\left(0.075 \mathrm{~min}^{-1}\right)\end{array}$ \\
\hline$k_{4}$ & $\begin{array}{l}\text { precursor to metabolite reverse grey matter rate } \\
\text { constant }\left(0.0056 \mathrm{~min}^{-1}\right)\end{array}$ \\
\hline 0 & denotes operation of convolution \\
\hline SG & specific gravity of brain tissue $(1.032 \mathrm{~g} / \mathrm{ml})$ \\
\hline CAL & $\begin{array}{l}\text { PET scanner to well counter calibration constant } \\
(\text { PET ct } / \mathrm{min} / \text { pixel }) /(\text { well } \mathrm{cts} / \mathrm{min} / \mathrm{ml})\end{array}$ \\
\hline D & duration of PET scan (min) \\
\hline
\end{tabular}

$$
\mathrm{LCBF}=\frac{6000}{\mathrm{SG}} \frac{Q^{*}(T)+\frac{1}{\lambda} \int_{0}^{T} Q^{*}(t) \mathrm{d} t}{\mathrm{CAL} \int_{0}^{T} C^{*}{ }_{\text {in }}(t) \mathrm{d} t-\frac{1}{p} \int_{0}^{T} Q^{*}(t) \mathrm{d} t},
$$

where $\mathrm{LCBF}$ is given in units of $\mathrm{ml} / \mathrm{min} / 100 \mathrm{~g}$ tissue. SG is the specific gravity of brain as in table 3. CAL is the calibration between the PET scanner and the continuous flow coincidence detectors (PET cts/min/

Table 3

Definition of symbols in ${ }^{18}$ F-FDG model 
Table 4

Normal LCMRG rates $(\mathrm{mg} / \mathrm{min} / 100 \mathrm{~g}$ tissue)

\begin{tabular}{lcccccc}
\hline & Cerebellum & $\begin{array}{l}\text { Temporal } \\
\text { cortex }\end{array}$ & $\begin{array}{l}\text { Visual } \\
\text { cortex }\end{array}$ & $\begin{array}{l}\text { Left } \\
\text { hemisphere }\end{array}$ & $\begin{array}{l}\text { Right } \\
\text { hemisphere }\end{array}$ & $\begin{array}{c}\text { Whole } \\
\text { brain }\end{array}$ \\
\hline Mean [7] & 4.65 & 5.12 & 6.56 & 4.58 & 4.54 & 4.59 \\
Standard deviation & 0.53 & 1.17 & 0.97 & 0.87 & 0.83 & 0.85 \\
Coefficient of variation & $11.5 \%$ & $22.9 \%$ & $14.8 \%$ & $19.0 \%$ & $18.1 \%$ & $18.5 \%$ \\
\hline
\end{tabular}

pixel $) /(\mathrm{cts} / \mathrm{min} / \mathrm{ml}) . \lambda$ is the decay constant for $0-15$ $\left(0.341 \mathrm{~min}^{-1}\right) . p$ is the blood:brain partition coefficient (0.9). $C_{\text {in }}^{*}(t)$ is the ${ }^{15} \mathrm{O}-\mathrm{H}_{2} \mathrm{O}$ arterial blood concentration and $Q^{*}(t)$ is the PET scanner count rate (PET cts/min/pixel).

\section{Results and discussion}

Maximum patient throughput is presently four scanning sessions per week. As computer analysis procedures become more automated, the expected throughput will double. Some 200 studies have been performed to date that involve PET scans of patients with epilepsy, Huntington's chorea, stroke, glioma brain tumors, Alzheimer's disease, spasmodic torticollis and other movement disorders. Normal volunteers are also studied to provide a database of normative local cerebral metabolic and blood flow values for comparison with the same brain regions of interest from the patients. Analysis of variance and other statistical tests are performed on the parametric values for each patient population.

Table 4 depicts local cerebral glucose metabolic rates for seven normal subjects. LCMRG for three primary areas of the brain are presented. The first is associated with control and coordination of voluntary muscle movements (cerebellum), the second reflects the intact grey matter cortical regions (temporal cortex) which may be related to memory, fine motor movements etc. and the last region is responsible for decoding visual images (primary visual cortex). In virtually all cases, the visual cortex depicts the highest glucose utilization rates. The cerebellum shows the lowest metabolic rates. The hemispherical averages do not differ significantly from each other nor do they differ from the cerebellar values. Severe depression of local glucose rates indicate loss of neuronal function. Stroke patients often have LCMRG values in the lesion area of $1-2 \mathrm{mg} / \mathrm{min} / 100 \mathrm{~g}$. Rarely do values exceed the visual cortex rates. If this occurs, it is usually in the superior frontal lobes and results from anxiety. The values for the coefficient of variation are typical of any physiological parameter determined even by the most accurate means. These values are near the expected average of $20 \%$.

Studies involving measurement of $\mathrm{LCBF}$ depict a coupling of LCMRG with blood flow. Decreased LCBF follows decreased LCMRG in normals, increased LCBF tracks increased LCMRG. However, in stroke patients, decreased LCMRG indicates neuronal loss. Blood flow initially decreases but then increases after a short period and eventually returns to normal values.

\section{Conclusions}

Positron emission tomography is an exciting field that permits the investigator to peer into the working human brain and examine numerous biological parameters without disturbing normal equilibrium physiology. PET measures regional brain function that cannot be determined by any other means including $C T$ and NMR imaging. Our understanding of brain physiology will increase as the technology advances yielding higher resolution instruments, new radiopharmaceuticals and thorough analysis of the existing PET data.

\section{References}

[1] M. Reivich, D. Kuhl, A. Wolf, J. Greenberg, M.E. Phelps, T. Ido, V. Casella, J. Fowler, E.I. Hoffman, A. Alavi, P. Som and L. Sokoloff, Circ. Res. 44 (1979) 127.

[2] L. Sokoloff, M. Reivich, C. Kennedy, M.H. Des Rosiers, C.S. Patlak, K.D. Pettigrew, O. Sakurada and M. Shinohara, J. Neurochem. 28 (1977) 897.

[3] J.C. Mazziotta, M.E. Phelps, J. Miller and D.E. Kuhl, Neurology 31 (1981) 503.

[4] E.J. Hoffman, S.C. Huang and M.E. Phelps, J. Comput. Asst. Tomogr. 3 (1979) 299.

[5] T. Ido, C.N. Wan, J.S. Fowler and A.P. Wolf. J. Org. Chem. 42 (1979) 2341.

[6] M.E. Phelps, S.C. Huang, E.F. Hoffman, C. Selin, L. Sokoloff and D.E. Kuhl, Ann. Neurol. 6 (1979) 371.

[7] The Cyclotron Corporation, 950 Gilman St., Berkeley, CA 94710 , USA.

[8] Engineering Note, LBL No. LS0806, serial no. M 5381.

[9] Digital Equipment Corporation, 146 Main St., Maynard, MA 01754, USA.

[10] Analogic Corporation, Audubon Rd., Wakefield, MA 01880 , USA.

[11] Gould Inc., Imaging and Graphic Division, 1870 Lundy Ave., San Jose, Ca 95131, USA.

[12] Computer Design and Applications, Inc., 411 Waverly Oaks Rd., Waltham, MA 02154, USA.

[13] M. Bergstrom, L. Eriksson, C. Bohm. G. Blomquist and J. Litton, J. Comput. Assist. Tomogr. 7 (1983) 42. 